- ACORN Australan College peroperative nuRses | JOURNAL OF PERIOPERATIVE NURSING

Volume 34 | Issue 2

Article 6

$5-30-2021$

\title{
Identifying barriers to patient advocacy in the promotion of a safety culture: An integrative review
}

Follow this and additional works at: https://www.journal.acorn.org.au/jpn

Part of the Perioperative, Operating Room and Surgical Nursing Commons

(c) (i)

This work is licensed under a Creative Commons Attribution 4.0 License.

\section{Recommended Citation}

Shoemark, Teena and Foran, Paula (2021) "Identifying barriers to patient advocacy in the promotion of a safety culture: An integrative review," Journal of Perioperative Nursing: Vol. 34 : Iss. 2 , Article 6.

Available at: https://doi.org/10.26550/2209-1092.1126

https://www.journal.acorn.org.au/jpn/vol34/iss2/6

This Article is brought to you for free and open access by Journal of Perioperative Nursing. It has been accepted for inclusion in Journal of Perioperative Nursing by an authorized editor of Journal of Perioperative Nursing. 


\section{Authors}

Teena Shoemark

MCN (Anaesthetics and Recovery Nursing), RN, MACORN

Dr Paula Foran

PhD, RN, FACORN, FACPAN, MACN

\section{Identifying barriers to patient advocacy in the promotion of a safety culture: An integrative review}

\begin{abstract}
Problem identification

Promoting patient safety, through patient advocacy, is an important part of the perioperative nurse role. However, identified barriers to effective patient advocacy have also reflected deficits in the characteristics of safety culture. This integrative review aims to highlight these barriers and discuss strategies for promoting patient safety within the perioperative context by presenting links between patient advocacy and safety culture.
\end{abstract}

\section{Literature search}

An electronic search of the databases, EBSCOhost, Academic search ultimate, Cumulative Index Nursing and Allied Health Literature (CINAHL), Healthsource, MEDLINE and PubMed, was undertaken and yielded ten articles for inclusion. Primary research included in this review consisted of five qualitative studies, three quantitative studies and two case studies. Further literature was used to provide background into this subject and guidance on writing this paper.

\section{Data evaluation synthesis}

The selected research was critically appraised for methodological quality using JBI critical appraisal checklists for case reports, qualitative and prevalence research. A data extraction table was used to record, group, compare and inform the integrative process of thematic analysis and data synthesis, generating themes that emerged through the selected literature.

\section{Implications for practice}

Synthesised findings will highlight the importance of patient advocacy by the perioperative nurse to increase patient safety. This review of the literature will present barriers to patient advocacy and discuss the suggestion that the key to greater patient safety may be an organisational commitment to enhance patient advocacy by perioperative nurses allowing them to speak up on behalf of their patients.

Keywords: patient safety, safety culture, patient advocacy, perioperative nursing 


\section{Introduction}

Patients put their trust in the health care system to provide high-quality, safe care that will meet their needs and expectations ${ }^{1}$. Acceptance of accountability for practice and acknowledgement of the nurse's role in protecting a patient's autonomy and right to care that is of high quality, and both clinically and culturally safe, is paramount ${ }^{2}$. This could not be more important than in the perioperative context, where patients are exposed to the vulnerabilities associated with undergoing anaesthesia when they are temporarily unable to act on their own behalf ${ }^{3}$.

In the words of Virginia Henderson, a famous nursing theorist, when defining patient advocacy in nursing

the nurse is temporarily unconsciousness, the love of life for the suicidal, the leg of the amputee, the eyes of the newly blind, a means of locomotion for the infant, knowledge and confidence for the young mother, and a 'mouthpiece' for those too weak or withdrawn to speak $^{4}$ p.63.

The objectives of this review are to understand the relationship between patient advocacy and safety culture in the perioperative context; to present the perioperative nurse role in patient advocacy; discuss some of the barriers to patient advocacy, including hierarchy in the perioperative environment and fear of blame; and identify strategies to overcome these barriers, including flattening the hierarchy, opencommunication and non-punitive approaches to risk reporting.

\section{Problem identification}

Patient advocacy in the perioperative context has been widely researched over the last two decades. Results have shown that perioperative nurses view their role as a protector from harm and a human rights activist ${ }^{3}$. Patient advocacy provides nurses with the opportunity to exercise their professional, moral and ethical perspective, promoting empowerment and professional satisfaction ${ }^{5}$. Barriers to perioperative nurse advocacy, such as hierarchy and communication constraints, have been well described in discussion papers reflecting on clinical practice ${ }^{6-8}$. These papers also highlight the relationship between advocacy and the concept of safety culture $^{6-8}$. As recognised by the Australian Commission on Safety and Quality in Health Care (ACSQHC), safety culture is a key element in the collaborative delivery of high-quality, safe care and is demonstrated through organisational attitudes that shape the behaviours of clinicians and leaders 9 . Despite this, the role of patient advocate has been impeded; therefore, identifying and overcoming the barriers to patient advocacy by perioperative nurses is vital for patient safety ${ }^{3}$.

\section{Literature search}

\section{Search strategy}

An electronic database search of the literature was conducted. Included in the search were, PubMed and, via EBSCOhost, Academic search ultimate, Cumulative Index Nursing and Allied Health Literature (CINAHL) complete, Healthsource: nursing/academic edition, MEDLINE, and MEDLINE complete.

Key terms used in the search were 'patient', 'advocacy', 'perioperative', 'operating room', 'nurs*', 'patient advocacy' and 'safety culture'. The PubMed MeSH and PubMed search builder were utilised to include medical subject headings (MeSH) terms in the search. MeSH terms were, 'patient safety'[Mesh],
‘Perioperative Care/ethics'[Mesh], 'Perioperative Care/legislation and jurisprudence'[Mesh], 'Perioperative Care/organsisation and administration'[Mesh]. Boolean phrases, AND and OR were applied to narrow the search terms and the truncation '*' applied to include plurals of key terms.

\section{Inclusion and exclusion criteria}

To access the most up-to-date primary research and scholarly, peer-reviewed literature, the search results were limited to the years 2015 to 2020 and articles from peerreviewed journals only. Included articles referenced the key terms and were in English due to language constraints of the authors. Exclusion criteria included research not related to nursing, patient advocacy or safety culture; secondary sources of research; protocols; guidelines and research not transferrable to the perioperative context.

\section{Data evaluation synthesis}

\section{Data extraction and evaluation}

Data extraction included the author, date of publication, origin of the study, population and sampling method, study design, level of evidence, key findings and limitations. In agreeance with Whittemore and Knafl, the diversity in research design of the included studies indicated the appropriateness for the application of quality appraisal tools ${ }^{10}$. Reliability and validity of the selected research was determined using the levels of evidence as described by Jirojwong, Johnson and Welch from level I, the highest, to level VII, the lowest ${ }^{11}$. The selected research was critically appraised for 'methodological quality' using the JBI critical 
appraisal checklists for case reports, qualitative and prevalence research ${ }^{12}$. Each checklist had between eight and ten questions that was allocated a score, 'yes', 'unclear', 'no', 'not applicable', which was interpreted by the author with a rating of low-, moderate- or high-quality research.

\section{Data analysis and synthesis}

As suggested by Whittemore and Knafl, the integrative review method was followed to analyse and synthesise the data through thematic analysis ${ }^{10}$. Data reduction initially involved grouping the research by study design. The data extraction table, used to record extracted data to be later used for comparison, is included as supplemental material. Data display was achieved through the applied table by grouping similar data. Comparison of the grouped data was used to generate themes and connections. The themes and connections were integrated for discussion and synthesised for verification and to draw conclusions.

\section{Descriptive findings}

As indicated in the PRISMA flow diagram (Figure 1), the search strategy identified 347 articles and four articles were identified through a search of the reference lists in the selected literature, as recommended by Liberati et $\mathrm{al}^{13}$. After duplicates were removed from the total 351 articles, 163

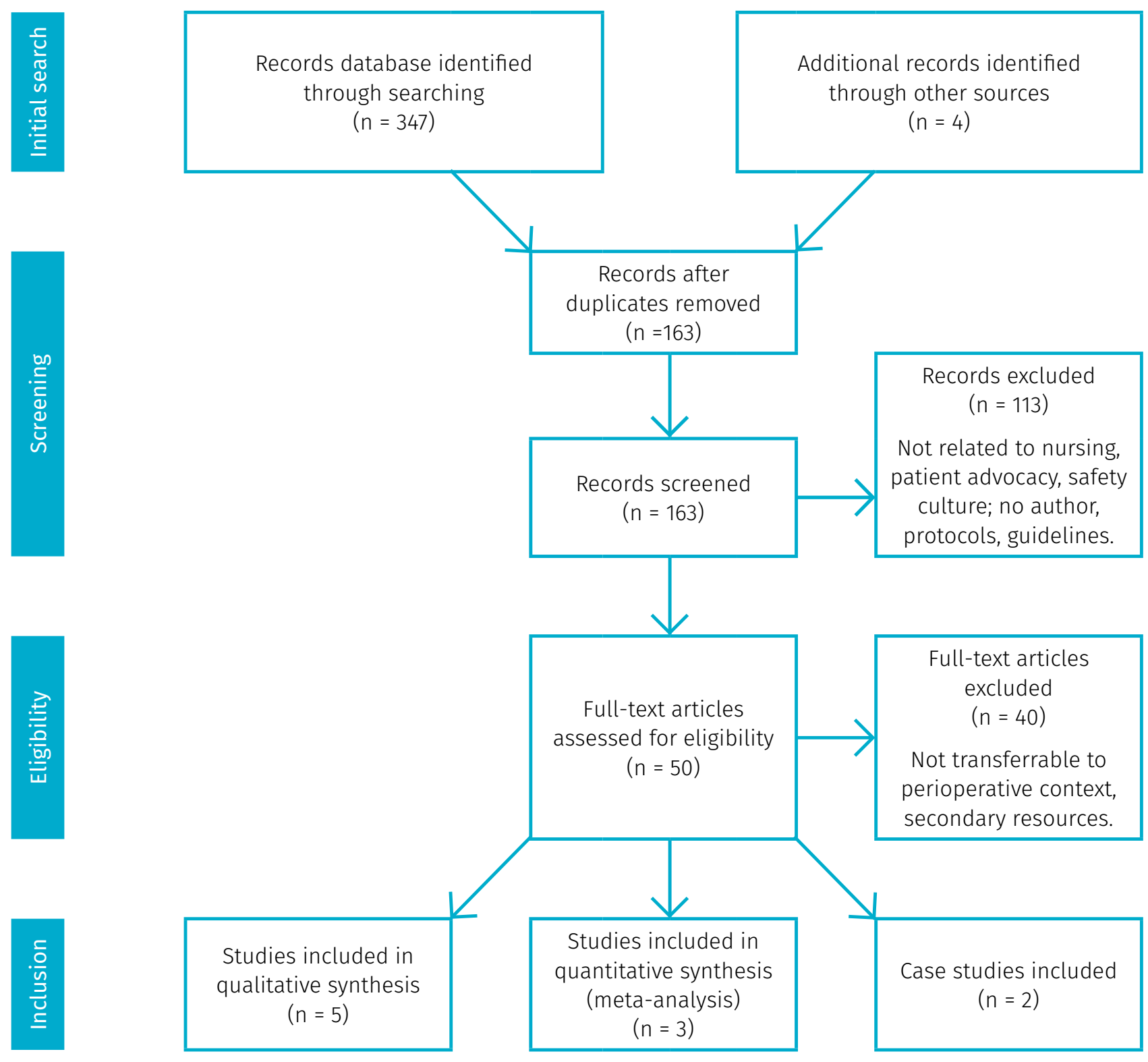

Figure 1: PRISMA flow diagram of papers for inclusion 
remained. Of the 163 articles, 113 articles were excluded after titles and abstracts were screened against the inclusion and exclusion criteria. The 50 remaining full-text articles were screened for relevance to the review aims resulting in 40 articles being excluded. The majority of the excluded full-text articles were found to be relevant to patient advocacy and safety culture; however, based on the recommendations of Jirojwong et al.11 and Joanna Briggs Institute (JBI) ${ }^{12}$ they were excluded as they were determined by the author to be lowlevel discussion papers or low-quality case reports where reliability and validity of the results could not be determined. Three full-text articles were also found to be relevant to the review aims but were excluded as they were review articles. A total of ten primary research articles were selected for inclusion. The origin of selected articles were the USA $(n=7)$, Sweden $(n=1)$, Australia $(n=1)$ and Canada $(n=1)$.

\section{Quality assessment}

The ten included articles were assessed for quality according to Jirojwong et al.11 and $\mathrm{JBI}^{12}$. The three quantitative studies were found to be level III-3 cross-sectional studies and were critically appraised across nine criteria for prevalence research to be of moderate quality. Quality was reduced by low response rates and description of sampling methods. The five level VI qualitative studies included four that consisted of semi-structured interviews and focus groups, and one with an etic approach that was observational with informal interviews. All qualitative studies included field notes or journaling, coding and thematic analysis. The five qualitative studies were critically appraised across ten criteria for qualitative research and found to be of high quality. Of the two level IV case studies, one was critically appraised against eight criteria and one against six of the eight criteria, due to no applicability to the study. Both were found to be of high quality.

\section{Results}

The analysis of the data was mapped through comparison for similarities in the methodology, aims and findings of the selected research. Through this iterative process the overarching theme to emerge linking perioperative patient advocacy and safety culture, was 'perioperative nurse role in patient advocacy'. Subthemes generated under barriers to perioperative advocacy were 'hierarchy in the perioperative environment' and 'fear of blame'.

\section{Discussion}

\section{Perioperative nurse role in patient advocacy}

Four qualitative studies researched the perspective of perioperative nurses as patient advocates ${ }^{14-17}$. Two qualitative studies found that perioperative nurses viewed their patients as vulnerable and that attentiveness to patients' needs and expectations builds trust and promotes advocacy ${ }^{14,15}$. Through the collective experiences of preoperative nurses, and endusers of preoperative care, Malley et al. found that there is often a gap between patient expectation and specialist knowledge that can negatively impact transitions of care $^{14}$. This study found that patients coming in for surgery expected that all the information pertaining to their care would be available when they arrived ${ }^{14}$. When gaps in information occur, distrust and fear builds, and patient outcomes are negatively impacted $^{14}$. This research revealed that nurses perceived themselves as important in filling these gaps to build trust, and that by gathering all the necessary information nurses put themselves in a position to protect their patients from harm ${ }^{14}$. Echoing these sentiments, Ingvarsdottir et al. reiterated the importance of these findings, explaining that while there was limited time preoperatively to spend with patients, perioperative nurses identified this as being very important to filling the gaps in information and building patient trust $^{15}$.

Sundqvist et al. highlighted that, despite the limited time perioperative nurses have with a conscious patient during transition to the operative phase, trust building was still achievable through acts of advocacy that promote psychosocial support, integrity and autonomy ${ }^{16}$. For example, in one study a nurse was observed to be conscious of meeting the patient's needs in addressing the patient by name, checking for comfort, assisting with transfer onto the theatre table, talking the patient through steps in the process and pulling the blinds down to cover the window into the theatre ${ }^{16}$. During the phase of anaesthesia, where the patient was unaware, members of the theatre team were also seen to protect the patient through constant surveillance, collaborative interactions, acts of informationsharing at different points in the patient's transition through the perioperative environment and challenging each other on decisions in patient care ${ }^{16}$

The findings of challenging decisions and surveillance for patient protection are also consistent with a qualitative study by Bacon that researched the nurse experience of 'failure-to-rescue' (FTR) postoperatively ${ }^{17}$. Although the concept of FTR is not specific to the perioperative context, the results are generalisable through application to current Australian standards 
in recognising the deteriorating patient ${ }^{18}$. It was found that the participants in this study viewed their role as patient protectors through patient surveillance in relation to their abilities to escalate care ${ }^{17}$. This was linked to patient advocacy, as the study highlighted both the importance and the difficulties in speaking up on behalf of the patient when deterioration is detected ${ }^{17}$. Through rich description, it was also found that junior nurses, in particular, have difficulty in knowing when or how intensely to pursue escalations of care when they have concerns for a patient's welfare ${ }^{17}$. FTR, even with experienced nurses in the PACU and despite efforts to advocate and escalate care for their patients, has resulted in adverse events ${ }^{19}$.

\section{Barriers to perioperative advocacy}

\section{Hierarchy in the perioperative environment}

Rich data from two qualitative studies found that the ability to escalate care is often associated with fear ${ }^{15,20}$. In a phenomenological study, the lived experience of participants described the view of being 'unpopular' for speaking up in the best interest of patients ${ }^{15}$. A grounded theory study found that culture in the perioperative environment was characterised by a 'steep hierarchy' that played a central role in the functioning and mood of the environment ${ }^{20}$. Although this study was of surgical resident doctors working in the operating suite, it was relevant in its insight into the culture of the perioperative milieu ${ }^{20}$. The experience of participants was described as avoiding conflict with both nurses and consultant doctors, suppressing feelings or using questioning, either indirect or direct, to challenge decisions ${ }^{20}$. This is consistent with the findings of
Rainer and Schneider that suggest that nurses feel subordinate to doctors, hindering their ability to speak up ${ }^{21}$. In support of this notion, one case study described a nurse raising concern with a surgeon over the viability of a written consent and, despite the nurse's concern, the surgeon insisting the patient still be transferred to the operating suite $^{22}$. Feeling pressured by the conflict between their professional obligations to the patient and the perspectives of the surgeon, the nurse transferred the patient to the theatre; however, the nurse did escalate her concerns to the manager ${ }^{22}$. The manager pursued the nurse's concerns with the surgeon, thus supporting the nurse and flattening the hierarchy, and the patient's surgery was subsequently postponed until a valid consent was obtained $^{22}$.

\section{Fear of blame}

Fear of blame was a common barrier to risk reporting within the selected literature ${ }^{15,20,23-25}$. The data from one quantitative study showed that 59 out of 352 participants revealed they had not reported a patient safety concern, with 33 of those citing the reason as fear of blame ${ }^{24}$. In the same study, data revealed that even though 94.8 per cent of participants believed their facility was supportive of risk reporting, 37 per cent did not report an unsafe practice they had $\operatorname{seen}^{24}$. One qualitative study also found that low rates of reporting risks to patient safety was due to a lack of opportunity for formal, open discussion and a fear of documented risk reports being used to leverage individual blame ${ }^{15}$.

\section{Links to safety culture}

Fan et al. hypothesised that surgical site infection rates were linked to the concept of safety culture ${ }^{25}$. This research used a survey with twelve dimensions of safety culture that examined perceptions of open communication, feedback, risk reporting processes and approaches, management of and support for patient safety, and teamwork ${ }^{25}$. Findings $(r=-0.90 ; \mathrm{Cl}$ $95 \%=[-0.45,0.99])$ revealed that poor organisational commitment to safety leads to low perceptions of safety culture by staff in the workplace, which in turn leads to higher rates of surgical site infections ${ }^{25}$.

In a quality improvement case study, Lozito et al. had identified an increase in patient harm from surgical error and 'near-miss' events that were often not being reported ${ }^{23}$. It was identified through a staff survey, that open communication and non-punitive approaches to risk reporting needed improvement ${ }^{23}$. In this study the implementation strategy included education for safety culture, standardising the reporting process and debriefing following reporting to promote open discussion and reflective learning ${ }^{23}$. This study showed that implementing strategies to improve organisational commitment to patient safety improved 'near-miss' reporting, with statistically significant results $(p=<0.05)^{23}$. Lozito et al. showed that an organisational commitment to safety, through improved communication strategies, resulted in a 15 to 20 per cent increase in staff satisfaction with aspects of safety culture - open communication, feedback, 'non-punitive' approaches to risk reporting and education ${ }^{23}$.

These findings are supported by a quantitative study that explored how safety culture influences team behaviour. The study found a statistically significant correlation between patient advocacy in 'speaking up' and a positive safety culture $(p=0.000)^{21}$. This study showed that a safety culture which is supportive of questioning, risk 
reporting and the ability to challenge on behalf of patient safety issues, reduced 'moral distress' experienced by nurses through promoting their ability to 'speak up' thus advocating for the safety of their patients ${ }^{21}$.

\section{Implications for perioperative nursing practice or research}

The aim of this integrative review was to understand the relationship between patient advocacy and safety culture, and identify strategies to promote patient advocacy and patient safety within the perioperative context. The included literature recognises the perioperative nurse role as a protector of patients from harm. The research highlights the complexities of the perioperative team environment and identifies hierarchical structures as a barrier to advocating for patient safety. Open communication and non-punitive approaches to risk reporting, were recognised as key characteristics of safety culture, greatly influencing the perioperative climate. For perioperative leaders, the findings of this review will provide context to the recently devised ACSQHC safety culture measurement toolkits, aimed at improving patient safety within Australian health care organisations ${ }^{9}$.

\section{Knowledge translation}

1. Perioperative nurses view patients within the perioperative environment as vulnerable, and themselves as protectors from harm. Through acts of advocacy, nurses execute their responsibility and moral compass to promote the rights of their patients and to provide the highest standard of safe patient care.

2. Nurses fearing to 'speak up' on behalf of their patients, when there is a perceived hierarchy and lack of support from clinical leaders, negatively impacts communication and promotes a poor safety culture.

3. Flattening the hierarchy through open communication strategies and non-punitive approaches to risk reporting were identified as promoting a positive safety culture that better supports patient advocacy.

\section{Limitations}

The results of this integrative review are limited by the low number of primary research articles found through the search strategy, with only one study being Australian. Generalisability and transferability of the results may be biased by only six of the included studies being specific to the perioperative context. Of those, only four could be related directly to Australian perioperative nursing practice.

\section{Conclusion}

This integrative review explored the perceptions of the perioperative nurse role in patient advocacy as protector from harm. Synthesised findings of the selected literature highlight that team culture can be a barrier to advocating for patient safety when it is hierarchical and promotes communication that is closed and punitive.

The ability of perioperative nurses to speak up on behalf of their patients is paramount in the operating suite where patients are vulnerable and often unable to speak for themselves. This advocacy sits close to the heart of perioperative nursing and perioperative nurses see this task as very important.

As limited literature was available on patient advocacy and safer patient outcomes, further research into these important links may be warranted.

The literature in this review revealed that strategies by organisational leaders to promote supportive, open communication, free from fear, have the potential to strengthen the ability of perioperative nurses as patient advocates, ultimately improving patient safety outcomes.

\section{Acknowledgment}

This paper was submitted to the University of Tasmania as part fulfilment of subject CNA803, Advanced Clinical Nursing Practice, for the Master of Clinical Nursing (Perioperative Nursing). The author sincerely wishes to thank Dr Paula Foran, unit coordinator, for her guidance throughout the master's course and work in preparing this paper for publication.

\section{References}

1. Australian Commission on Safety and Quality in Health Care (ACSQHC). The state of patient safety and quality in Australian hospitals 2019 [Internet]. Sydney: ACSQHC; 2019 [cited 2020 October 5]. Available from: www.safetyandquality.gov.au/sites/default/ files/2019-07/the-state-of-patient-safetyand-quality-in-australian-hospitals-2019.p df

2. Battie R, Steelman V. Accountability in nursing practice: Why it is important for patient safety. JPN 2016;29(4):11-14.

3. Munday J, Kynoch K, Hines S. Nurses' experiences of advocacy in the perioperative department: A systematic review. JBI Database System Rev Implement Rep 2015;13(8):146-189. DOI: 10.11124/ jbisrir-2015-2121.

4. Henderson, $\mathrm{V}$. The nature of nursing. AJN 1964; 64(8):62-68.

5. Sundqvist A-S, Holmefur M, Nilsson U, Carlsson A. Perioperative patient advocacy: An integrative review. J Perianesth Nurse 2016;31(5):422-433. DOI: 10.1016/j.jopan.2014 12.001.

6. McClelland G, Smith MB. Just a routine operation: A critical discussion. J Perioper Pract 2016;26(5):114-117. DOI: $10.1177 / 175045891602600504$. 
7. Clark C, Kenski, D. Promoting civility in the OR: An ethical imperative. AORN J 2017;105(1):60-66. DOI: 10.1016/j. aorn.2016.10.019

8. Crook C. Advocacy: How far would you go to protect your patients? AORN J 2016;103(5):522-526. DOI: 10.1016/j. aorn.2016.03.009.

9. Australian Commission on Safety and Quality in Health Care (ACSQHC). Patient safety culture [Internet]. Sydney: ACSQHC: 2019 [cited 2020 October 5]. Available from: www.safetyandquality.gov.au/our-work/ indicators-measurement-and-reporting/ patient-safety-culture.

10. Whittemore R, Knafl K. The integrative review: Updated methodology. J Adv Nurs 2005;52(5):546-553.

11. Jirojwong $\mathrm{S}$, Johnson $\mathrm{M}$, Welch, editors. Research Methods in Nursing and Midwifery: Pathways to Evidence-based Practice. 2nd ed. Melbourne: Oxford university press; 2014.

12. Joanna Briggs Institute (JBI). Critical appraisal tools [Internet]. Adelaide: JBI; 2020 [cited 2020 September 19]. Available from: https://joannabriggs.org/critical-appraisaltools.

13. Liberati A, Altman DG, Tetzlaff J, Mulrow C, Gøtzsche PC, loannidis JPA, et. al. The PRISMA statement for reporting systematic reviews and meta-analyses of studies that evaluate health care interventions: Explanation and elaboration. PLoS Med 2009; 6(7): e1000100. DOI: 10.1371/journal. pmed.10000100.
14. Malley A, Kenner C, Kim T, Blakeney B. The role of the nurse and the preoperative assessment in patient transitions. AORN J 2015;102(2):181e1-181e9. DOI: 10.1016/j. aorn.2015.06.004

15. Ingvarsdottir E, Halldorsdottir S. Enhancing patient safety in the operating theatre: From the perspective of the experienced operating theatre nurses. Scan I Caring Sci 2017;32(2):951-960. DOI: 10.1111/scs.12532.

16. Sundqvist A-S, Holmefur M, Nilsson U, Carlsson A. Promoting person-centred care in the perioperative setting through patient advocacy: An observational study. I Clin Nurs 2018;27(11-12):2403-2415. DOI: 10.1111/ jocn.14181.

17. Bacon C. Nurses' experiences with patients who die from failure to rescue after surgery. J Nurs Scholarsh 2017;49(3):303-311. DOI: 10.1111/jnu.12294.

18. Australian Commission on Safety and Quality in Health Care (ACSQHC). Recognising and responding to acute deterioration standard [Internet]. Sydney: ACSQHC; 2017 [cited 2020 September 30]. Available from: www.safetyandquality.gov.au/standards/ nsqhs-standards/recognising-andresponding-acute-deterioration-standard.

19. State Coroners Court of New South Wales (NSW). In: Coroners Court of New South Wales, editor [Internet]. Sydney: State Coroners Court; 2019 [cited 6 May 2021]. Available from: www.coroners.nsw.gov.au/ coroners-court/coronial-findings-search. html?searchtext=p.\%201-31.\&searchYear=2019.
20. Bould D, Sutherland S, Sydor D, Naik $\checkmark$, Friedman Z. Residents' reluctance to challenge negative hierarchy in the operating room: A qualitative study. I Can Anesth 2015;62(6):576-586. DOI: 10.1007/ s12630-015-0364-5.

21. Rainer J, Schneider J. Testing a model of speaking up in nursing. JONA 2020;50(6):349354. DOI: 10.1097/NNA.00000000000000896.

22. Gilbert J, Gillespie B. Surgical consent and the importance of a substitute decisionmaker: A case study. JPN 2017;30(1):15-19.

23. Lozito M, Whiteman $K$, Swanson-Biearman, Barkhymer M. Good catch campaign: Improving the perioperative culture of safety. AORN I 2018;107(6):705-714. DOI:10.1002/aorn.12148.

24. Cole D, Bersick E, Skarbek A, Cummins K, Dugan K, Grantoza R. The courage to speak out: A study describing nurses' attitudes to report unsafe practices in patient care. $J$ Nurs Manag 2019;27:1176-1181. DOI: 10.1111/ jonm.12789.

25. Fan C, Pawlik T, Daniels T, Vernon V, Banks K, Westby $P$ et al. Association of safety culture with surgical site infection outcomes. I Am Coll Surg 2016; 222(2):122-128. DOI: 10.1016/. jamcollsurg.2015.11.008. 